\title{
The Development of an Automated Clean-up for Fat Extracts in the Routine Analysis of Organochlorine Compounds in Fish Meat
}

\author{
Ana-Andreea CIOCA $^{1 *}$, Olaf HEEMKEN ${ }^{2}$ and Marian MIHAIU ${ }^{1}$ \\ $1^{*}$ Department of Animal Production and Food Safety, University of Agricultural Sciences and Veterinary \\ Medicine, 3-5 Mănăștur Street, 400372, Cluj-Napoca, Romania. \\ ${ }^{2}$ Department of Organic Residues,LAVES- Institute for fish and fishery products, Schleusenstraße 1, \\ 27472, Cuxhaven, Germany. \\ *corresponding author: anaandreeacioca@yahoo.com
}

Bulletin UASVM Veterinary Medicine 74(1) / 2017,

Print ISSN 1843-5270; Electronic ISSN 1843-5378

DOI:10.15835/buasvmcn-vm: 12588

\begin{abstract}
The present work describes the development of a new, automatic High Performance Liquid Chromatography (HPLC) Clean-up step, in the methodology of sample preparation and multi-residue determination of organochlorine compounds (OCs) in fish meat. 24 OCswere taken into study. In addition 7 Polychlorinated Biphenyls (PCBs), 7 chlorobenzene compounds and one 2,3,7,8-Tetrachlorodibenzo-p-dioxin (TCDD) were investigated.The HPLC conditions were established in accordance with the validated traditional Clean-up step of the laboratory. The technique was applied on a dilution of analytes of interest in order to establish the period of time in which the compounds are eluted. Another set of experiments involved fish oil, in order to identify and separate the fat fraction from the analytes. To confirm the findings of the experiments mentioned above, extracts of fish samples obtained after Accelerated Solvent Extraction (ASE) were examined. The samples were spiked with the analytes of interest before HPLC clean-up step and quantified throughGas Chromatography coupled with tandem Mass Spectrometry (GC-MS/MS). A HPLC clean-up technique lasting 38 minutes/ sample was developed. The method is not suitablefor OCs such as Endosulfansulfat and Endrine Ketonedue to the very low recovery results.
\end{abstract}

Keywords: High Performance Liquid Chromatography Clean-up, fish, organochlorine compounds

\section{INTRODUCTION}

OCs are known to have a profound negative effect on the environment. Ingested by fish, through contaminated feed or other pesticide treatments, they are retained in the adipose tissue andcan determine the pollution of fish meat released on the market.The risk for humans is the chronic toxicity, following the ingestion of small quantities of pesticides in food (Aktar et al., 2009). These compounds focus their action on enzyme systems, on vitamins and hormonesand have carcinogenic activity (Roncati et al., 2016). Monitoring organochlorine pesticide residues is a current concern in the European Union. Analytical methods for the analysis of polychlorinated biphenyls (PCBs) and organochlorine pesticides (OCPs) are widely available and are the result of a vast amount of environmental analytical method development and research on persistent organic pollutants (POPs) over the past 30-40 years (Muir et al., 2006). Even so, the analysis of pesticide residues in fish samples is challenging due to the low concentrations and large number of analytes that need to be monitored and quantified in a complex matrix (Kaczyński et al., 2017, Rodrigues et al., 2016). Usually a solvent-based extraction of the sample is required in the first part of the pesticide determination process from which will result contaminants and unwanted matrices. This step is nearly always followed by an appropriate 
clean-up step to isolate the target analytes and remove unwanted matrix components (Sørensen et al., 2015). Some clean-up techniques such as the solid phase-extraction (SPE) using Florisil, alumina or silica, require more time and handling. Others such as gel permeation chromatography (GPC) - a subset of exclusion chromatography (SEC) are automated (David et al., 2017). Automated techniques are more likely to improve the productivity of the laboratories from the perspective of time and operational safety. In our case, the availability of a HPLC 0 instrument made it possible to experiment for the development of an automated clean-up for a small food safety and consumers' protection laboratory with few employees. Such a method could replace the validated method of SPE using silica gel of the

\section{AIMS AND OBJECTIVES}

he aim of the present study was to develop an efficient HPLC clean-up for fat extracts, in the routine analysis of mainly OCs in fish meat for the laboratory of Organic Residues, IFF, Cuxhaven. The objective was to validate a new method and to increase the productivity of the laboratory by replacing the manual labour with automatic means which require less time and surveillance.

\section{MATERIALS AND METHODS}

Reagents. N-hexane ROTISOLV® min. $99 \%$ Pestilyse andacetone ROTIPURAN ${ }^{\circledR}$ min. 99,8\% p.a. were purchased from Carl Roth (Germany). Dichloromethane for residue and pesticide analysis was purchased from AppliChem GmbH (Germany). Helium 5.0 and nitrogen 5.0 were purchased from Linde Gas (Germany). Pure certified analytical standards used for external and internal standards came from Sigma-Aldrich (Germany).Fish oil was provided by (San Omega GmbH (Berlin, Germany). The Gas Chromatography coupled with tandem Mass Spectrometry (GC-MS/MS) instrument used possessed a $700 \mathrm{GC} / \mathrm{MS}$ Triple Quadrupole and came from Agilent Technologies (USA).The provider of the HPLC column was Macherey\&Nagel (Düren, Germany).

Sample preparation. Three types of experiments were conducted. The first sample consisted in a dilution of the analytes of interest in $n$-hexane (2:3 ratio). The fractions (F) collected after HPLC clean-up were transferred in separate evaporation flasks. Each flask was evaporated until $1 \mathrm{ml}$ at $40^{\circ} \mathrm{C}$ and then transferred into $2 \mathrm{ml}$ vials for quantification through Gas Chromatography coupled with tandem Mass Spectrometry (GCMS/MS). The method applied was previously designed for the compounds taken into study and the software used was MS Quantitative Analysis. The second experiment was conducted on 100 mg fish oil as a fat matrix. We determined the weight of the collection flasks on the analytical scale Sartorius, (Germany) before the clean-up procedure. After the clean-up, we evaporated the solvents from the collection flasks using nitrogen gas. We calculated the differences between the final weight of the collection flasks and the empty flasks. In order to confirm the findings of the two experiments above, a third sample was analysed. Three extracts of native salmon samples obtained after freeze-drying and Accelerated Solvent Extraction (ASE), were spiked with the $50 \mu \mathrm{l}$ external standard (ESTD) before the HPLC clean-up. The ESTD consisted in the analytes of interest diluted in n-hexane and a concentration of $1 \mu \mathrm{l} / \mathrm{ml}$. The extracts were spiked with $100 \mu \mathrm{l}$ internal standard (ISTD) before ASE. The ISTD consisted of a 4, 4'-DDT (D8), Hexachlorbenzene (HCB13C6), Tetrachlornaphtalin (TCN) and PCB 198 at a concentration of $1 \mu \mathrm{l} / \mathrm{ml}$. After the cleanup, the collection flasks were transferred in one evaporation flask the samples were treated the same as the dilution of analytes of interest in n-hexane (2:3 ratio).

HPLC clean-up. For the HPLC clean-up we used a Nucleosil@ 100-7 silica gel particle size 7 column $\mu \mathrm{m}$, with the dimension: 250 length $\mathrm{x} 10$ $\mathrm{mm}$ inner diameter. In order to reproduce the manual steps in our new, automated technique, we have set the next parameters in the ChemStation software of our HPLC system with Diode Array Detection (HPLC-DAD) 1290 Infinity series, Agilent Technologies (USA):injection volume: $500 \mu \mathrm{l}$; solvents: n-hexane, dichloromethane and acetone arranged in a non-polar to polar gradient; flow: $6.000 \mathrm{ml} / \mathrm{min}$; stop time: $38 \mathrm{~min}$; thermostat temperature: $20^{\circ} \mathrm{C}$ between $0-15 \mathrm{~min}$, $40^{\circ} \mathrm{C}$ between $15-25$ min and $20^{\circ} \mathrm{C}$ between $25-38$ min; fraction collector: trigger mode time-based starting min 0 until min 30;time slices of fraction collector: 2 min. 


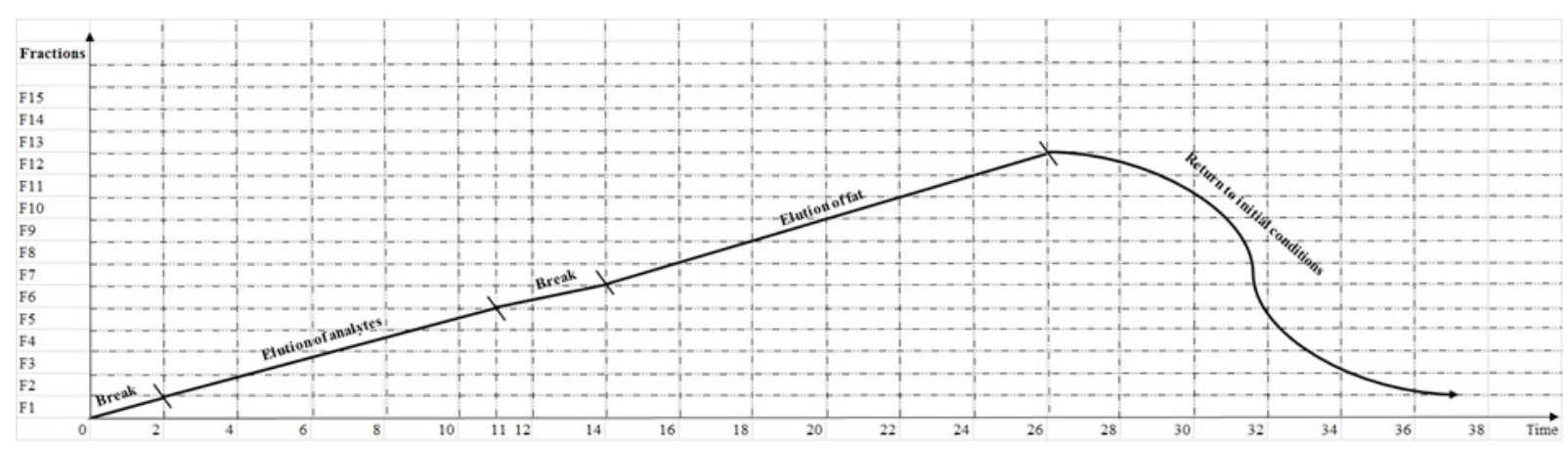

Fig. 1. The elution of analytes and fat during HPLC clean-up

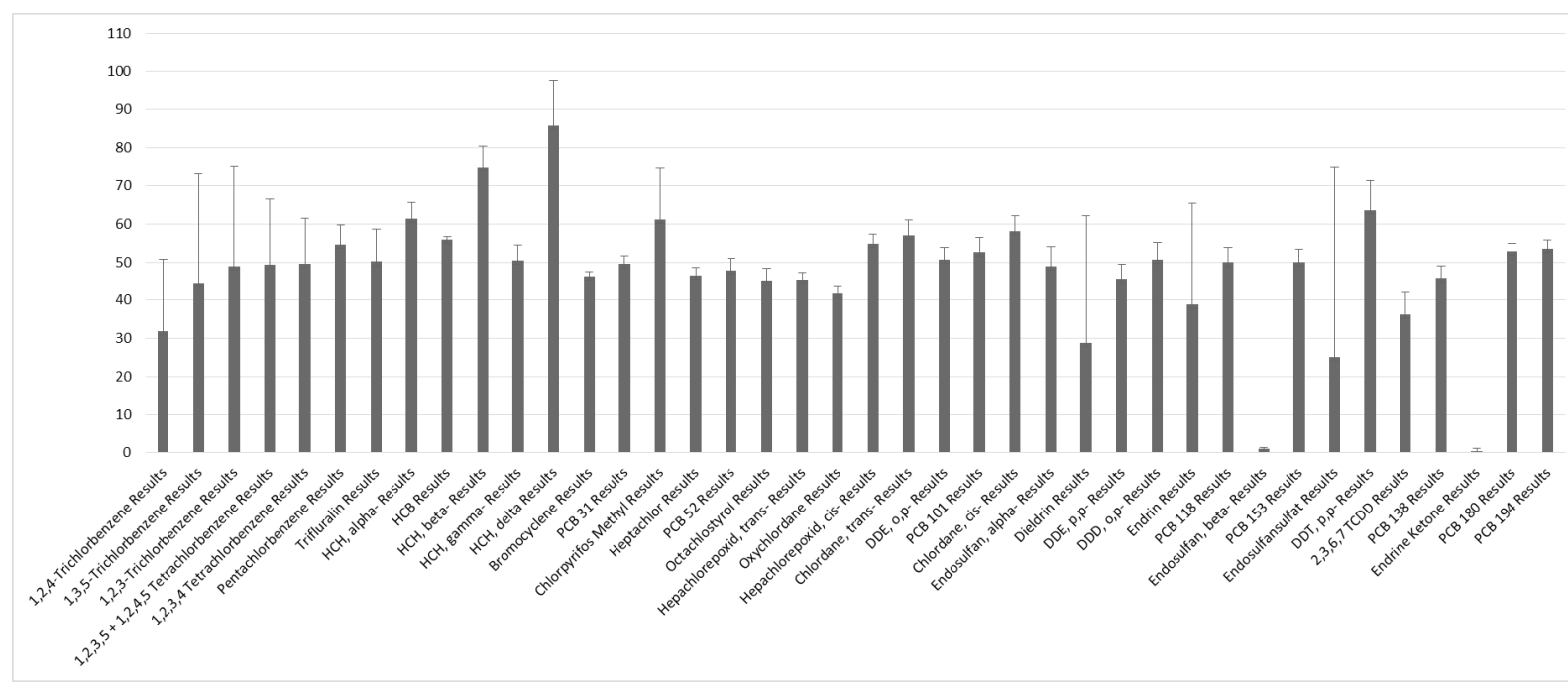

Fig. 2. Recoveries $(\%)$ of the analytes of interest in $(n=4)$ fish oil samples (mean \pm STDEV)

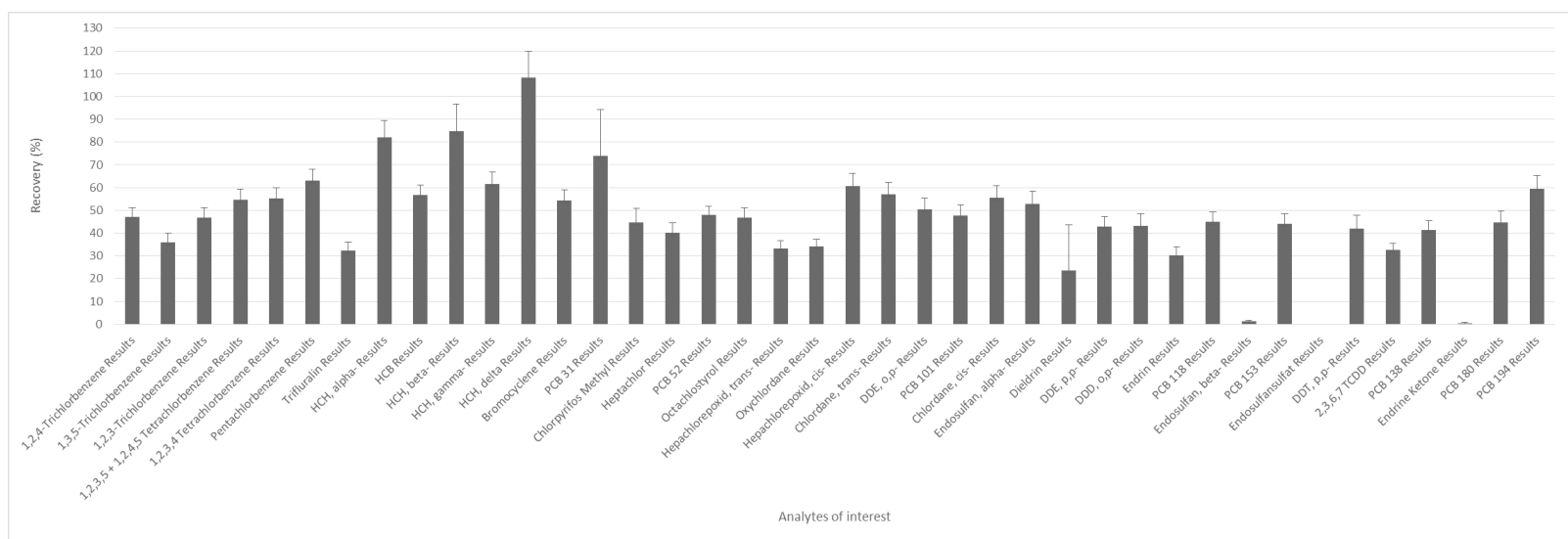

Fig. 3. Recoveries (\%) of the analytes of interest in ( $n=9)$ ASE extracts of salmon samples (mean \pm STDEV)

Data analysis. For the quantitation of absolute recoveries a four point calibration curve was prepared from the target analytes within the range 10-100 ng $\mathrm{mL}^{-1}$. These curves presented a good linearity $\left(\mathrm{R}^{2}\right.$ for all curves were $\left.<0.999\right)$. A good reproducibility of the samples (spiked at level 50 $\mathrm{ng} \mathrm{g}^{-1}$ ) was achieved through similar recoveries of the replicates ( $\mathrm{n}=9$ ASE extracts of fish samples) for each compound taken into study and standard deviations below $12 \%$. LOD was in the range of 1-6 $\mathrm{ng} \mathrm{g}^{-1}$ and 24-43 $\mathrm{ng} \mathrm{g}^{-1}$ for Octachlostyrol, DDE o.p, DDE p.p., and LOQ 3-18 ng g ${ }^{-1}$,respectively 72138ng g ${ }^{-1}$. 


\section{RESULTS AND DISCUSSIONS}

The first experiment conducted, revealed that the analytes of interest elute from min 2, F2, until min 10, F5. The elution took place under a non-polar solvent gradient, consisting of a higher percentage of $n$-hexane and a lower percentage of dichloromethane. No compounds eluted between min $0-2$, in F1 and nor between min 10-14, F5-F6. In the second experiment, the fat elution started at min 14, F7 and ended at min 26, F12. Numerous attempts showed an approx. $100 \%$ recovery of fat. In this time frame, the gradient moved from nonpolar to relative polar and polar solvents, namely dichloromethane and acetone. Considering the experiments above, the method was modified for the third experiment. Fractions were collected only from min 2, F2 until min 11, 1/2 F10. The time slices were changed to $3 \mathrm{~min}$, so the clean-up ended with 3 fractions. The method continued until minute 38 , with fat being sent directly into the waste container and out of the HPLC instrument. From min 24 until min 38 , the instrument was able to return to the initial conditions of the method (Fig.1.). The spiked fish oil samples salmon extracts revealed recoveries of the analytes of interest in the range of $25-85 \%$. Almost no values were recorded for Endosulfan beta and Endrine Ketone. The measurements made on spiked ASE salmon extracts showed recoveries in the range of 23-108\%. Besides confirming the low recoveries obtained by Endosulfan beta and Endrine Ketone, another compound has to be eliminated from the method. Endosulfansulfat has was not detected during measurement. Systematical errors or sample handling could be the reason of these problems. Even if the overall recoveries were not the ideal ones, the originality of this new clean-up protocol remains based on the concept that this method used automated, free and already present means of the laboratory. Even though numerous studies from literature describe other validated clean-up protocols with better recoveries of organochlorine pesticides (Chen et al., 2009, Rodrigues et al., 2016, etc, Thompson et al., 1989, etc), in our case, finding a proper alternative for the SPE using deactivated silica gel clean-up with a minimum of financial investments was desirable.

\section{CONCLUSION}

An automated HPLC clean-up was developed, efficiently separating the OCs, PCBs, chlorobenzene compounds and one dioxin (2,3,7,8-TCDD) taken into study. The result is a purified extract ready for GC-MS/MS quantification. Compounds such as Endosulfan beta, Endosulfansulfat and Endrine Ketone require more investigations. In this stage the method requires an optimization of the overall analytes recoveries, reduction of process time and solvent consumption.

Acknowledgement. We gratefully acknowledge the financial support from the German Federal Environment Foundation (DBU Osnabrück).

\section{REFERENCES}

1. Aktar MDW, Sengupta D, Chowdhury A (2009). Impact of pesticides use in agriculture: their benefits and hazards, Interdisciplinary Toxicology 2(1): 1-12.

2. Kaczyński P, ŁozowickaB, Perkowski M,SzabuńkoJ (2017). Multiclass pesticide residue analysis in fish muscle and liver on one-step extraction-cleanup strategy coupled with liquid chromatography tandem mass spectrometry. Ecotoxicology and Environmental Safety138:179-189.

3. Chen S, Yu X, HeX, Xie D, Fan Y, Peng J (2009).Simplified pesticide multiresidues analysis in fish by lowtemperature cleanup and solid-phase extraction coupled with gas chromatography/mass spectrometry. Food Chemistry 113 (4): 1297-1300.

4. David F, Devos C, Dumont E, Yang Z, Sandra P, Huertas-Pérez $J F(2017)$. Determination of pesticides in fatty matrices using gel permeation clean-up followed by GC-MS/MS and LC-MS/MS analysis: A comparison of low- and highpressure gel permeation columns. Talanta 165: 201-210.

5. Muir D, Sverko E (2006). Analytical methods for PCBs and organochlorine pesticides in environmental monitoring and surveillance: a critical appraisal. Anal Bioanal Chem. 386(4): 769-789.

6. Rodrigues ET, Pardal MA, Salgueiro-González N, MuniateguiLorenzo S, Alpendurada M.F (2016). A single-step pesticide extraction and clean-up multi-residue analytical method by selective pressurized liquid extraction followed by on-line solid phase extraction and ultra-high-performance liquid chromatography-tandem mass spectrometry for complex matrices. Journal of Chromatography A 1452: 10-17.

7. RoncatiL, Piscioli F, Pusiol T (2016). The endocrine disruptors among the environmental risk factors for still birth. Science of the total environment, 563-564: 1086-1087.

8. Sørensen L, Silva MS, Meier S, Booth AM (2015). Advances in miniaturization and increasing sensitivity in analysis of organic contaminants in marine biota samples. Trends in Environmental Analytical Chemistry 6-7: 39-47.

9. Thompson TS, Schellenberg DH, Naikwadi KP (1989). HPLC cleanup procedures for the determination of 2,3,7,8TCDD in fish tissue.Chemosphere19(91-6): 45-50. 